\title{
AUTOMATED WATER HEAD CONTROLLER FOR DOMESTIC APPLICATION
}

\author{
Santosh K. Verma ${ }^{1}$ \\ ${ }^{I}$ M. Tech (Instrumentation Engineering), Department of Electrical Engineering, National Institute of Technology, \\ Agartala
}

\begin{abstract}
In environmental terms, water is the lifeblood of the planet, still a huge amount of water is being wasted everyday because of overflow of water tanks installed in our houses. Though there are technologies to prevent this, but they fail to satisfy economical or reliability criteria because of which people don't want to use them. Here we have designed a cost effective, reliable system, which is easy to install and requires almost zero maintenance. In system design we have used ATmega8 (8-bit CPU) microcontroller manufactured by Atmel corporation and ultrasonic sensor module (HC-SR04) for contactless measurement of water level.
\end{abstract}

Keywords - Home Automation, AVR, Ultrasonic Sensor, Contactless Level Measurement, ATmega8, UART, Water Head Controller, HC-SR04

\section{INTRODUCTION}

In the process of measurement of distance using ultrasonic sensor, sensor transmits a train of pulse and waits for it to receive at receiver end after reflecting from object. If there will be any object in front of the sensor then the train of pulse will be exactly received at the receiver end. Time taken between transmitted pulse and received pulse is the time taken by sound wave (Ultrasonic) to travel from transmitter to object and back to the receiver. Measuring this time we can find the distance of the object from the sensor module [1].

\section{BLOCK DIAGRAM}

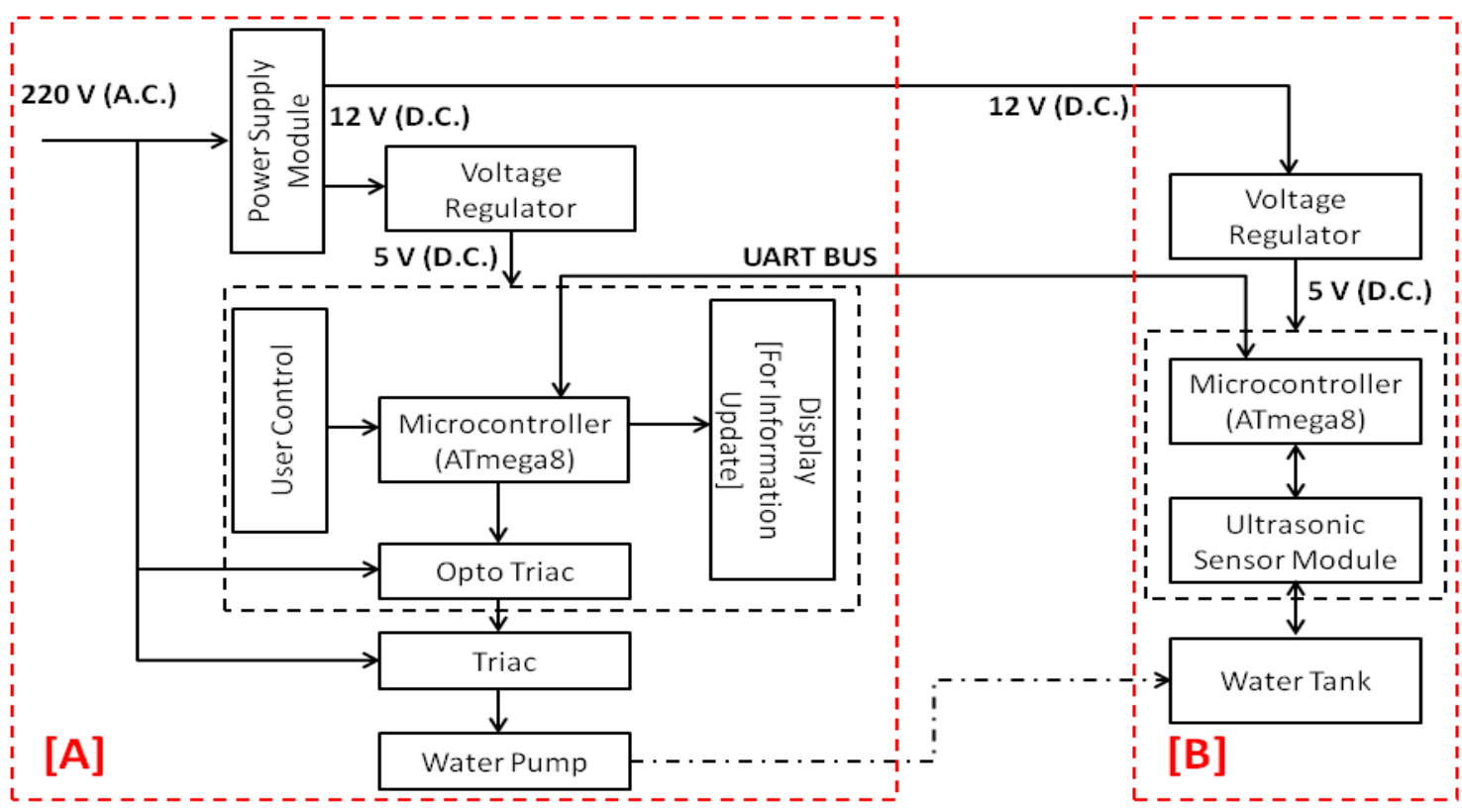

Fig.1. System Block Diagram
Let time taken is $\mathbf{t}$ seconds and considering speed of sound in air $340 \mathrm{~m} / \mathrm{s}$ [1]. If the distance of the object is $\mathbf{D}$ in meters Then,

$2 \mathrm{D}=340 \mathrm{t}$

$$
\mathrm{D}=\mathbf{1 7 0 t}
$$

So by measuring time $\mathbf{t}$, we can easily find the distance between the sensor and the object i.e. water level in this case. 
The block diagram of the whole system is divided into two parts, $\mathbf{A}$ and $\mathbf{B}$. $\mathbf{B}$ is the part of system consisting of ultrasonic sensor module (HC-SR04) so it needs to be installed with the water tank where $\mathbf{A}$ is the part which we can install anywhere as per our own convenience near the water pump as it consists of user control and display unit for the information update (level of water in the tank). Both parts $\mathbf{A}$ and $\mathbf{B}$ of system are connected with 3-wires:

A. $\mathbf{V}_{\mathbf{C C}}(12$ V D.C. $)$

B. Ground

C. Data bus or UART BUS (used for bidirectional data transfer): Both microcontrollers can communicate over this bus using UART protocol.

\section{SYSTEM DESIGN}

The whole system is mainly divided in two parts PART A and $\mathrm{B}$ (refer to block diagram), one is installed with water tank and another near water pump. So the implementation of the whole system can be explained in following steps:

\subsection{System Design of Part A:}

This part of system mainly consisting of an ATmega8, 8-bit microcontroller for the processing and controlling, user control part consisting of controls for various operation i.e. to run system in manual mode, display unit for updating of information to the user i.e. water level in the tank, MOC3041 zero crossing Optotriac to provide isolation between high voltage and control logic and a BTA16, 16 Amp Triac to run the water pump. The circuit diagram of PART A is shown below:

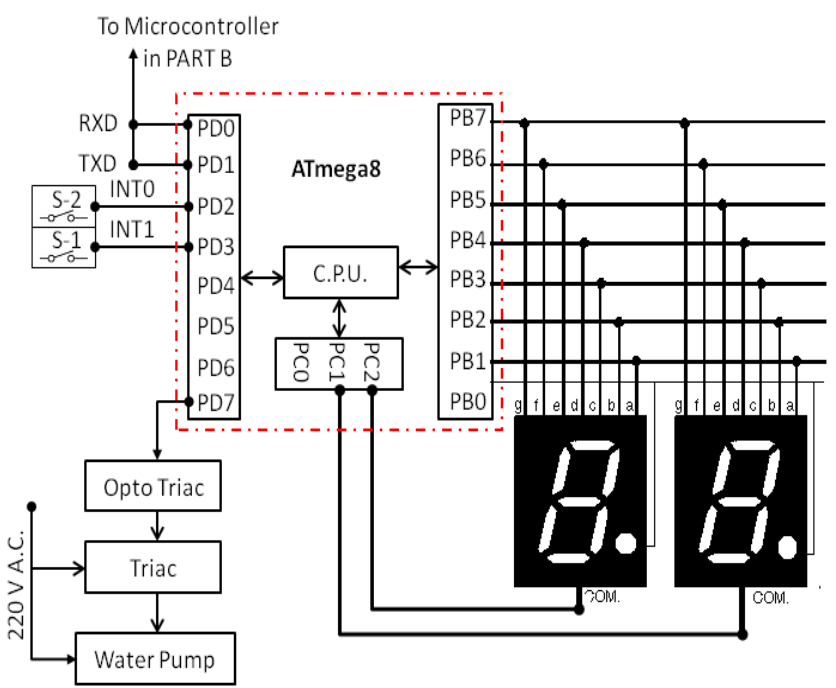

Fig.2.Circuit Diagram of PART A

Here in the circuit diagram PB, PD and PC stands for PORT $\mathrm{B}$, PORT D and PORT $\mathrm{C}$ and number in front indicates their location at PORT. Here power connection is not shown for simplicity, they should be considered connected to respective pins. All pins used here except PD0, PD1 PD2, PD3 are used for normal I/O PORT operation.

ATmega8 has a UART channel operates in full duplex mode. Its RX and TX are connected to PD0 and PD1 [2], so here these pins are used to receive and transmit the data using UART Protocol. We can connect two CPUs using UART protocol as per the diagram below:

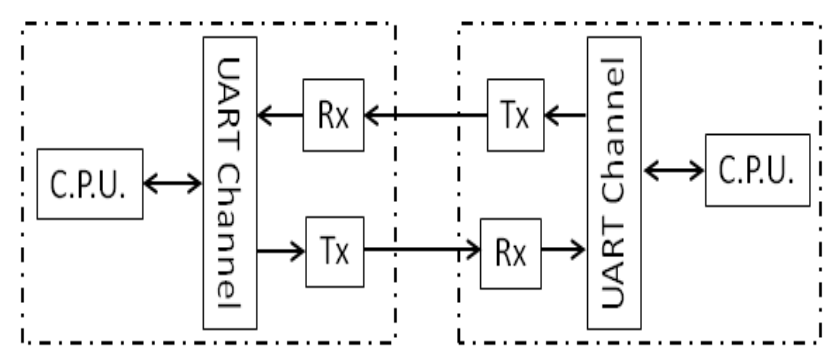

Fig.3. CPU connection using UART

As we can see in the picture above that it uses two wires for bidirectional data transfer. In our system we are using UART to stabilise the communication between two CPUs of PART $\mathrm{A}$ and $\mathrm{B}$ and as both parts are installed at different locations, it can be costly and complex to use two wires for bidirectional data transfer. To make it economical and simple system is designed in such a way that it requires only single wire for bidirectional data transfer for that we have connected both CPUs as per diagram below:

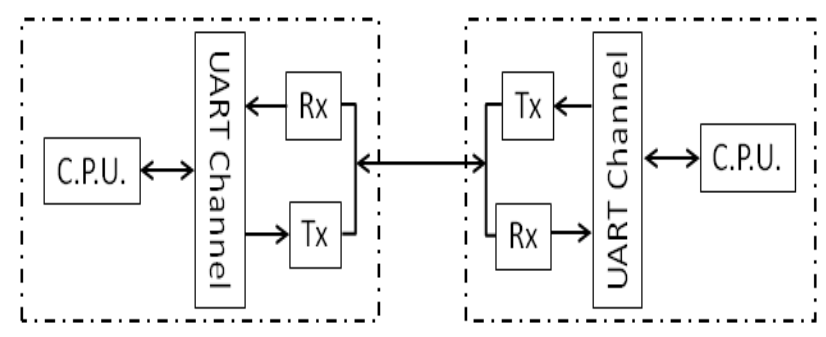

Fig.4. CPU connection using single wire UART

To connect both CPUs from part A and B in our system (see block diagram) over a single wire; CPU of part A is programmed to act as master and another one as Slave. To avoid any type of data collision over bus, only Master can initiate any type of communication. In the beginning we turn on TX of master and RX of the slave so that master can send data to slave and slave can receive it. When master needs any information from slave i.e. water level in the tank, it sends command to the slave and switches OFF its TX and switches $\mathrm{ON}$ its RX so that it can receive the data from the slave. Once slave receives the command it switches OFF its RX and switches ON its TX so that it can send the data to the master. On the completion of this cycle they restore their initial status i.e. master with TX $O N$ and slave with $R X O N$. In this way bidirectional data transfer takes place over a single wire using UART Protocol. The same may be understood from the picture below: 


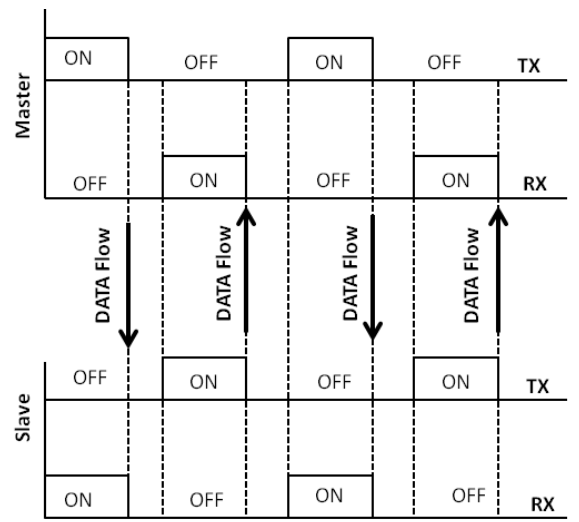

Fig.5. Algorithm Diagram of single wire UART

In user control section we have provided two switches S-1 and S-2 connected to PD3 (INT1) and PD2 (INT0) of ATmega8. They are used to generate an interrupt request externally when pressed. S-2 is used to run system in setup mode for the one time measurement of variables used for the determination of quantities; this is explained in next section briefly. Functioning of S-1 depends on the status of S-2 as explained below:

S-2 not pressed: Use S-1 to start water pump manually irrespective of water level in the tank. Stopping will be still automatic.

S-2 is pressed: When S-2 is pressed system enters in the set up mode for the one time measurement of variables used for the determination of quantities. In setup mode press S-1 to start the water pump manually and press again to stop it.

To provide information of water level in the tank to the user here two 7-segment displays are used which are connected at PORT B with their data bus and with PC1 and PC2 for their display control.

To drive the water pump from ATmega8 we need some switching equipment capable of switching $220 \mathrm{~V}$ A.C. supply from 5 V TTL logic. We can use electromechanical relay or solid state relays. Solid state relays have various advantages over electromechanical relays so in our application we have also used solid state relays to drive water pump from control circuit. We have used BTA16, 16 Amp Triac by ON Semiconductor comes in TO-220 package capable of block voltage up to $800 \mathrm{~V}$ and capable of control current up to 16 Amp [3].

We are using our control system to switch $220 \mathrm{~V}$ supply so we need to protect it from any overvoltage, for that we have used MOC3041 zero crossing OptoTriac. It isolates our control system from high voltage line and save system from damage because of any over voltage.

\subsection{System Design of Part B:}

This Part of system is mainly consisting of an ATmega8, 8bit microcontroller and a HC-SR04 ultrasonic sensor module. The work of this part is to measure the water level in the tank and provide the measured value to the Part $\mathrm{A}$ when requested. A image of HC-SR04 ultrasonic sensor module is shown below:

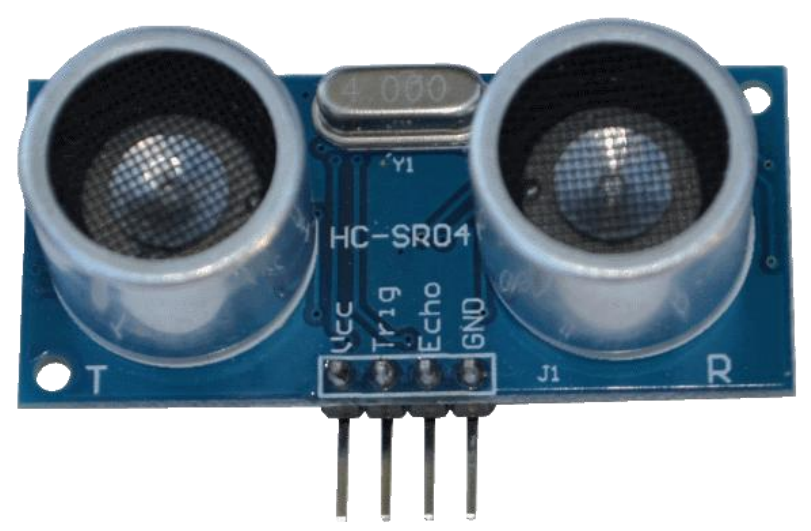

Fig.6. Ultrasonic Sensor Module (HC-SR04) [4]

It is capable of measuring distance $2 \mathrm{~cm}$ to $450 \mathrm{~cm}$ with precision of $0.3 \mathrm{~cm}$, operated from $5 \mathrm{~V}$ D.C. and consumes $15 \mathrm{~mA}$ of current [5] which makes it perfect for our application. This sensor module has 4 pins, their name and working is illustrated below:

$\mathbf{V}_{\mathbf{C C}}$ : Used to power up module with 5 V D.C. Supply.

GND: Used to connect with ground or with $0 \mathrm{~V}$ of power supply.

Trigger: When a high level pulse is applied at this pin at least for $10 \boldsymbol{\mu S}$ then module initiates the transmission of train of pulse at its transmitter.

ECHO: Once we trigger the module to initiate the transmission of train of pulse, module set the echo pin at high level and on the reception of train of pulse module set it back at low level. This change in voltage level at echo pin is also called as ECHO signal. Time between the rising and falling edge of the voltage level on the echo pin is the time taken to travel from transmitter to object and back to receiver as explained in the picture below:

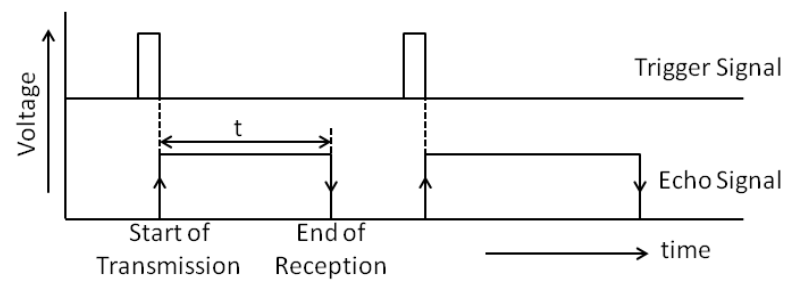

Fig.7. Output Waveform at Echo Pin (Echo Signal)

So here our aim is to measure the time $\mathbf{t}$ of echo signal as shown in picture above. So before explaining how this can be measured with ATmega8 microcontroller let's look at the connection diagram of ultrasonic sensor module with ATmega8. 


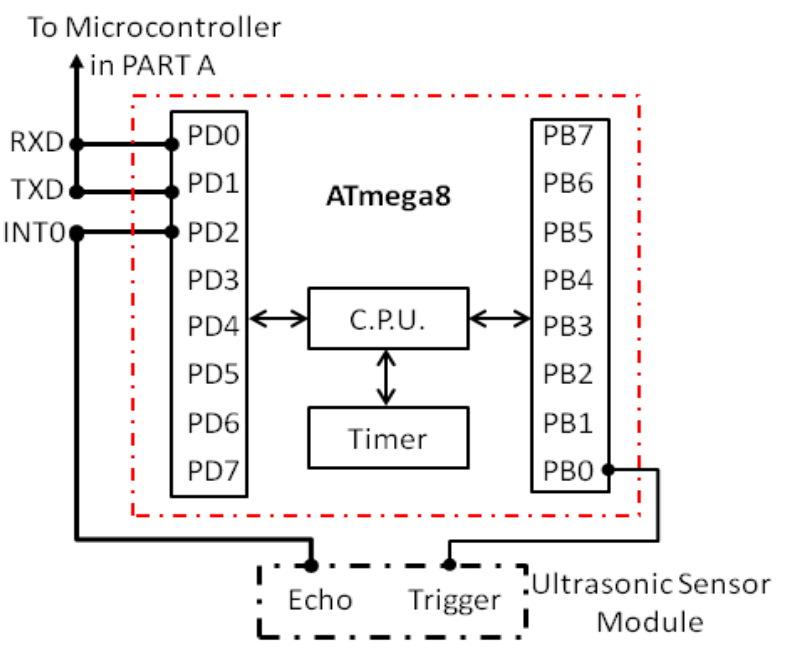

Fig.8. Circuit Diagram of PART B

In circuit diagram PD and PB stands for I/O PORT B and D i.e. PORT D, PORT B and number represents their location at PORT. In circuit diagram power supply connections to both ATmega8 and sensor module is not shown for the simplicity. They should be considered connected at their respective pins.

Here we are using PB0 for normal I/O operation while PD2 is used to generate software interrupt externally at each rising and falling edge of voltage signal applied i.e. echo signal. Atmega8 has an inbuilt 16 bit timer/counter named with timer1 which we have used here for the measurement of time t. Timer is running at $2 \mathrm{MHz}$, in upcounting mode.

Measurement of time t: First set PB0 at high level for a small duration (more than $10 \boldsymbol{\mu S}$ ) to trigger the sensor module to initiate the transmission of train of pulse at its transmitter setting the echo pin at high level. This will generate an interrupt request in atmega 8 at rising edge. While executing the interrupt handler we programmed it to reset the timer/ counter value to zero. As soon as the train of pulse will be received at its receiver, it will set the echo pin at low level. This will request an interrupt at falling edge of echo signal. While executing the interrupt handler we have programmed it to capture the timer value in the variable which will be used for the evaluation of time $\mathbf{t}$. This is also explained in the figure below:

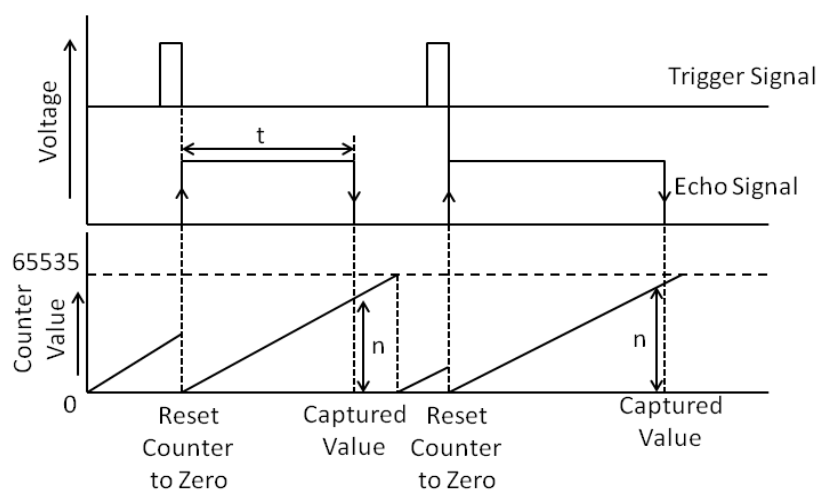

Fig.9. Measurement of time $t$ by ATmega8
We have stated earlier that timer is programmed to run at 2 $\mathrm{MHz}$ then time taken to increment counter value by 1 will be the time period $\mathbf{T}$ of the timer.

So, $\mathrm{T}=1 /\left(2 * 10^{6}\right)$

Here $\mathbf{T}$ is in seconds. Now let's assume, captured value of timer is $\mathbf{n}$ then,

$$
\mathbf{t}=\mathbf{n} * \mathbf{T}
$$

By using this equation we can measure time $\mathbf{t}$ (in seconds) from captured counter value of timer.

Substituting this value of " $t$ " in equation (1):

$\mathrm{D}=170 * \mathrm{t}$

$$
D=170 * n^{*} \mathbf{T}
$$

Using equation (3) we can calculate the distance D (in meters) between the sensor and the object. Now we have calculated the distance of water level from the sensor module but we need to represent it with respect to height of water tank (all distances in the picture below are in meter).

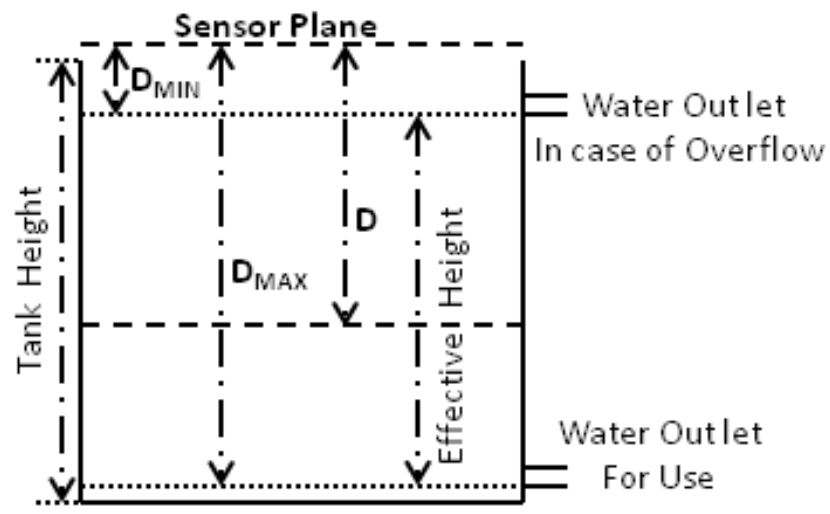

Fig.10. Various Heights in Water Tank

Distance of an object is measured from the horizontal plane of sensor module. Practically we cannot fill the water up to horizontal plane of sensor module as it has some minimum detection range. So we need to install the sensor module little bit higher than the maximum water filling capability of tank, this distance from the plane of sensor module is represented by $D_{\text {MIN }}$. In same manner there will be a maximum distance from sensor module; this is represented by $D_{\mathrm{MAX}}$ in the picture.

So our range of operation in terms of height of water tank will be $\mathrm{D}_{\text {MIN }}$ to $\mathrm{D}_{\text {MAX. }}$.

Now let's say at some instant water level is at distance D from the plane of sensor then effective height (in percentage) of water level in the tank given by:

Effective height $(\%)=\left(\left(D-D_{M I N}\right) /\left(D_{M A X}-D_{M I N}\right)\right)^{* 100}$ 
So percentage height of water is calculated from this equation and displayed at 7-segment display for user information.

Measurement of $\mathbf{D}_{\mathrm{MIN}}$ and $\mathbf{D}_{\mathrm{MAX}}$ : To calculate the effective height of water level in water tank from equation-4 we need to know the values of $D_{\text {MIN }}$ and $D_{\text {MAX }}$ before. These values can be measured by the system itself during the first time setup. We have provided a switch $(\mathrm{S}-2)$ in the user control section of PART A (refer to the System design of PART A) pressing switch S-2 system will enter into setup mode. Now to measure $\mathrm{D}_{\mathrm{MIN}}$ fill the tank up to its full capacity and press $\mathrm{S}-2$ again to measure the value of $\mathrm{D}_{\mathrm{MIN}}$. For the calculation of $D_{\text {MAX }}$ empty the tank and press $S-2$ to measure the value of $D_{\text {MAX. }}$. Once the values of $D_{\text {MIN }}$ and $D_{\text {MAX }}$ are calculated they will be stored in the EEPROM of ATmega8 for the further use. Pressing S-2 again will exit the setup mode and system will start operating in normal mode.

\section{WORKING OF DEVICE}

Device can be operated in following two modes:

\section{A. Automatic Mode}

B. Manual mode

Working of device in both modes is explained as below: Automatic Mode: In automatic mode the system will be controlled by the microcontroller. System will keep monitoring the water level in the tank; once the water level will fall below the $20 \%$ of the effective height, it will automatically start the Water Pump and store the running status in the EEPROM of microcontroller for permanent storage. Once the tank will be filled with water, water pump will be automatically turned off. In case of power failure system will resume from its last state i.e. if the motor was running before power failure then once the power will resume system will automatically start the water pump irrespective of water level in the tank and will stop as soon as tank will be filled.

Manual Mode: In some cases we may need to start the pump irrespective of the water level in the tank (in automatic mode water pump start automatically, when water level fall below $20 \%$ of the effective height) for that we have provided a switch in the user control section, pressing the switch will turn on water pump and start filling the tank. Once tank will be filled with water, system will automatically switch off the water pump. Here also in case of power failure system will resume from its last state i.e. if the motor was running before power failure then once the power will resume system will automatically start the water pump irrespective of water level in the tank and will stop as soon as tank will be filled.

Here I want to mention that system has manual control to switch on the water pump but not to turn it off (turning off water pump is fully automated for the normal operation. We can switch off the water pump manually only when system is in setup mode) as we don't need any manual stopping of the system. If in any case we need to stop the water pump manually user just needs to switch off the power switch of the power supply module and it will halt the whole system or put the system in setup mode by pressing S-2 and then press S-1 twice to stop.

\section{PRODUCT IMPLEMENTATION PLAN (PDRD)}

Note: Cost is estimated in Indian Currency.

\begin{tabular}{|l|l|l|l|l|l|}
\hline Name of Component & Quantity & Specification & Purchased from & Cost/Unit & Total \\
\hline Ultrasonic Sensor Module & 1 & HC-SR04 & $\underline{\text { www.evelta.com }}$ & 199 & 199 \\
\hline ATmega8 & 2 & 8-bit CPU & $\underline{\text { www.evelta.com }}$ & 76 & 152 \\
\hline Triac & 1 & 16 Amp-TO-220 Package & www.evelta.com & 38 & 38 \\
\hline Opto Triac & 1 & $\begin{array}{l}\text { MOC3041 } \\
\text { (Zero crossing Opto Triac) }\end{array}$ & www.digibay.in & 19 & 19 \\
\hline Heat Sink & 1 & & www.onlinetps.com & 10.5 & 10.5 \\
\hline Power Supply Module & 1 & 12V, 100 mA & Local Market & 40 & 40 \\
\hline 7-Segment Display & 2 & $\begin{array}{l}0.5 \text { inch } \\
\text { (Red, Common Anode) }\end{array}$ & www.evelta.com & 6.5 & 13 \\
\hline Voltage Regulator & 2 & 7805 & www.evelta.com & 7.95 & 15.9 \\
\hline miscellaneous & As required & Resistor, Capacitor, Switch & www.evelta.com & 10 & 10 \\
\hline & & & & Total & 497.4 \\
\hline
\end{tabular}

Here we have not included the cost of PCB designing and manufacturing as it vary with both quality and quantity. Cost estimated here is required to build a single unit but if we build more than 1000 units or go for mass production then per unit cost can be reduced below Rs. 200/-

\section{CONCLUSION}

In this paper, process of manufacturing of a very low cost, reliable and easy to install automated water head controller system based on ATmega8, 8 bit microcontroller from Atmel Corporation and HC-SR04 Ultrasonic sensor module has been presented. If we manufacture and install it within our houses then we can save a huge amount of fresh water and electricity wastage every day. 


\section{REFERENCES}

[1] Made Saraswati, Endrowednes Kuantama, Pono Mardjoko, "Design and Construction of Water Level Measurement System Accessible Trough SMS," $6^{\text {th }}$ UKSim/AMSS European Symposium on Computer Modeling and Simulation (EMS), 2012, pp. 48,53, 14-16 Nov. 2007.

[2] “ATmega 8/L Datasheet,". [Online]. Available: www.atmel.com/images/atmel-2486-8-bit-avrmicrocontroller-atmega8_1_datasheet.pdf

[3] "BTA16 Datasheet,". [Online]. Available: http://www.onsemi.com/pub_link/Collateral/BTA16 -600CW3-D.PDF

[4] "HC-SR04 Datasheet,". [Online]. Available: http://www.micropik.com/PDF/HCSR04.pdf

[5] "HC-SR04 Datasheet,". [Online]. Available: http://www.micropik.com/PDF/HCSR04.pdf 